This item was submitted to Loughborough's Research Repository by the author.

Items in Figshare are protected by copyright, with all rights reserved, unless otherwise indicated.

\title{
Effective and efficient preparation for the unforeseeable
}

PLEASE CITE THE PUBLISHED VERSION

http://www.incose.org/symp2016/

\section{PUBLISHER}

(c) The Author(s). Permission granted to INCOSE to publish and use

\section{VERSION}

AM (Accepted Manuscript)

\section{PUBLISHER STATEMENT}

This work is made available according to the conditions of the Creative Commons Attribution-NonCommercialNoDerivatives 4.0 International (CC BY-NC-ND 4.0) licence. Full details of this licence are available at: https://creativecommons.org/licenses/by-nc-nd/4.0/

\section{LICENCE}

CC BY-NC-ND 4.0

\section{REPOSITORY RECORD}

Hinsley, Steven W., Michael Henshaw, and Carys E. Siemieniuch. 2019. "Effective and Efficient Preparation for the Unforeseeable". figshare. https://hdl.handle.net/2134/21127. 


\title{
Effective and Efficient Preparation for the Unforeseeable
}

\author{
S.W. Hinsley \\ Loughborough University \\ Engineering Systems-of-Systems Group, \\ Wolfson School of Engineering, \\ Loughborough University, Leicestershire, \\ LE11 3TU, UK \\ s.w.hinsley2@lboro.ac.uk
}

\author{
Prof. M.J Henshaw \\ Loughborough University \\ Engineering Systems-of-Systems Group, \\ Wolfson School of Engineering, \\ Loughborough University, Leicestershire, \\ LE11 3TU, UK \\ m.j.d.henshaw@lboro.ac.uk
}

\author{
Prof. C.E. Siemieniuch \\ Loughborough University \\ Engineering Systems-of-Systems Group, Wolfson School of Engineering, Loughborough \\ University, Leicestershire, LE11 3TU, UK \\ c.e.siemieniuch@lboro.ac.uk
}

Copyright @ 2015 by S. W. Hinsley. Published and used by INCOSE with permission.

\begin{abstract}
This paper hypothesizes that a System-of-Systems (SoS) that is not fit-for-purpose is so because it cannot implement the correct, timely and complete transfers of Material, Energy and/or Information (MEI) between its constituents and with its external environment that are necessary to achieve a particular result. This research addresses the problem of maintaining a SoS fit-for-purpose after unpredictable changes in operation, composition or external factors by creating a method, implemented as an engineering process and supported by an analysis technique to enhance the affordance [ "Features that provide the potential for interaction by "Affording the ability to do something” [1]\} of SoS constituents for MEI transfer and reveal potential undesirable transfers.
\end{abstract}

\section{Introduction}

This paper summarizes research addressing the problem of how to keep a System-of-Systems (SoS) fit-for-purpose. The choice of research topic was motivated by the author's observations from working in the defense industry with several companies over a career of 30 plus years. There appeared to be a tendency for delivered products and services that, although meeting their requirements, needed modification to maintain a desired capability from the composing SoS, and hence be made fit-for-purpose. Changes in the SoS situation, for example the operational environment, requirement or the SoS capability components often rendered the SoS unfit for purpose due to a combination of the two reasons below in various proportions:

- The SoS capability was degraded and could no longer bring about the desired outcome for which it was designed.

- The SoS needed to provide some different functionality to that for which it was designed in order to achieve the desired outcome.

Causes of fit-for-purpose loss were dynamic and varied, often due to situational changes dictating that modifications to SoS constituent systems, to recover SoS fitness-for-purpose, had 
to be made close to, or at the point of utilization, frequently by the personnel working as part of the SoS using "work-arounds". A work-around is an engineering solution that is sufficient but rarely optimal in terms of efficiency or cost. Systems engineering shows that corrective action is most effectively and economically done early in the lifecycle, but it is acknowledged that total avoidance of late-stage modifications is unfeasible, which posed the question "What could be done by suppliers to facilitate maintenance of fitness-for-purpose?” It may be noted that one of the characteristics of a SoS noted by Maier [2] is that it is evolutionary; this implies that development of a SoS always requires the adaptation of an existing (legacy) set of systems: there is no clear sheet.

\section{Re-Configurability}

The Need for Re-configurability. In his holistic approach to risk management Hopkin [3], notes that risk is a "Circumstance, Action, Situation or Event (CASE) with the ability to impact key dependencies". Such impacts are equivalent to Systems-of-Systems not being fit-for-purpose. More generally, the SoS not being fit-for-purpose (i.e. able to do what the user requires) is often due to unforeseen circumstances, actions, situations or events, so that personnel working as part of the SoS have to modify the constituent systems close to the point of employment so that they can converge towards their aims. If the necessary system modifications are not feasible, the users' subsequent failure to achieve their objectives may have consequences ranging from increased costs to loss of life or property.

CASE that might adversely affect a SoS being fit-for-purpose can be categorized as "known", "known-unknown”, “unknown-known” and "unknown-unknown” [4]. Engineering actions can be taken to enhance fitness, and the better known these CASE are (i.e. the more predictable the CASE) the more directly they can be affected by engineering actions [5] such as design for robustness, resilience and re-configurability. Ring states "A system formalized by prescient design cannot respond to unforeseen situations."[6]. The realization of a systems capability is dependent on the simultaneous readiness of several components known as Lines of Development (LoD). The UK MoD's eight Defence Lines of Development are a typical example, being:

Training, Equipment, Personnel, Information, Doctrine, Organization, Infrastructure and Logistics.

It should be noted that this is not an exhaustive set of LoDs: it is likely that others such as Legal, Commercial and Finance will need to be considered.

Robustness and resilience can be designed-in to a system but any benefit they provide against unknown-unknown factors is largely due to serendipity: Ring and Tenorio, [7] state "A system formalized by prescient design cannot respond to unforeseen situations." The major LoD contributing to fitness-for-purpose maintenance in this circumstance (the focus of this work) is often the personnel working as part of the SoS. In support of this, General Sir Rupert Smith states "On every occasion that I have been sent to achieve some military objective in order to serve a political purpose, I, and those with me, have had to change our method and re-organize in order to succeed. Until this was done we could not use our force effectively. On the basis of my lengthy experience, I have come to consider this as normal - a necessary part of every operation” [8].

"Unknown-unknown” CASE poses a significant challenge, which needs to be addressed. Building on Ashby's work on "Requisite Variety" [9], Boardman \& Sauser [10] state "The uncertain and unknowable environment in which the SoS must operate presents a mystery of 
endless proportions, the only proper response to which is to have increasing variety, of a continually emerging nature, to deal with unforeseeable reality that eventually becomes clear and present danger”.

The "How, Where and Who" of Maintaining a SoS fit-for-purpose. SoS constituent and sub-system adaption has the potential to more effectively enhance MEI transfers at low levels than SoS re-configurability which has been widely utilized, because adaption actions are at a higher resolution and hence the adaption can be more closely tailored to address a changed circumstance. Re-configurability and adaptation are employed together in balance and proportion tailored to each individual case. The Fit-For-Purpose (FFP) method, process and technique facilitates SoS constituent system suppliers to equip their products and services affordably and conveniently with solution components, rather than solutions, to capitalize on the ingenuity and resourcefulness of utilizing personnel close to where they operate to efficiently and effectively address unforeseen changes when they occur. As Dalton commented, "But, ultimately, it is people who turn technology into capability; people who are experts in their profession with a comprehensive knowledge of the operational environment” [11]"

\section{SoS Constituents, Transfers and Affordances}

In 2008, the UK MoD defined Defense LoDs as "the elements that must be brought together to deliver military capability to operational users" and states that "In addition to the Defence LoDs, Interoperability is included as an overarching theme that must be considered when any Defence LoD is being addressed" [12].

System Engineering has been described as “The management of the emergent properties” [13]. Emergent properties are not attributable to one component of the system, so similarly systems and systems-of-systems engineering has a strong focus on the interactions between constituents, and accordingly this research has a focus on the interactions between the constituents of a SoS. At the fundamental level, these interactions are considered to be transfers of Matter, Energy and Information (MEI) Thus the designed operation of an instantiated SoS of interest is predicated upon the correct, timely and complete transfers of matter, energy and information between the SoS constituents to achieve the purpose(s) of the SoS. This research identifies additional Inherent and Independent Material, Energy and Information (MEI) Sources, Sinks and Bearers (SSBs) in a SoS constituent system that are not managed or captured by its defining documentation. These SSBs may cause undesirable emergent properties when integrated with other SoS constituents into a SoS, or may be exploited to enhance the affordance for MEI transfer to address shortfalls.

SSB structures able to transfer MEI are considered as affordances, defined by Sillitto at the INCOSE ASEC 2011 conference as "Features that provide the potential for interaction by "Affording the ability to do something, as perceived by the user, to achieve some goal” [14].

An illustration of the terms "Intended", "Inherent" and "Independent" used to describe MEI transfers and SSBs may assist the reader here. For example, a maritime surveillance radar system is the System of Interest (SoI). To electrically supply the radar control cabinet the designer specified an intended MEI (electrical energy) transfer from one of the ship's supplies to the cabinet by a Steel Wire Armored (SWA) cable. 

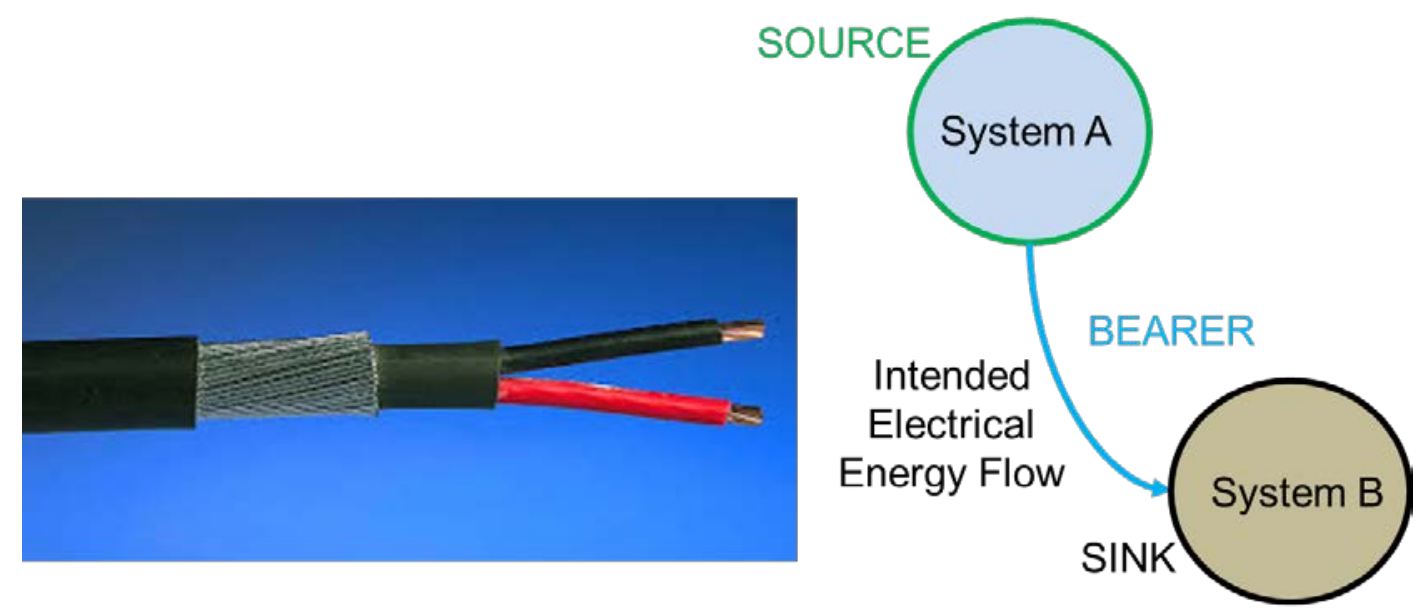

"Designed-For"

Figure 1: SWA DC power cable and Intended MEI Transfer (DC Electrical Energy)

This chosen bearer solution has inherent properties that enable it to conduct much more than DC electrical current however, as illustrated on the left of Figure 2 below.
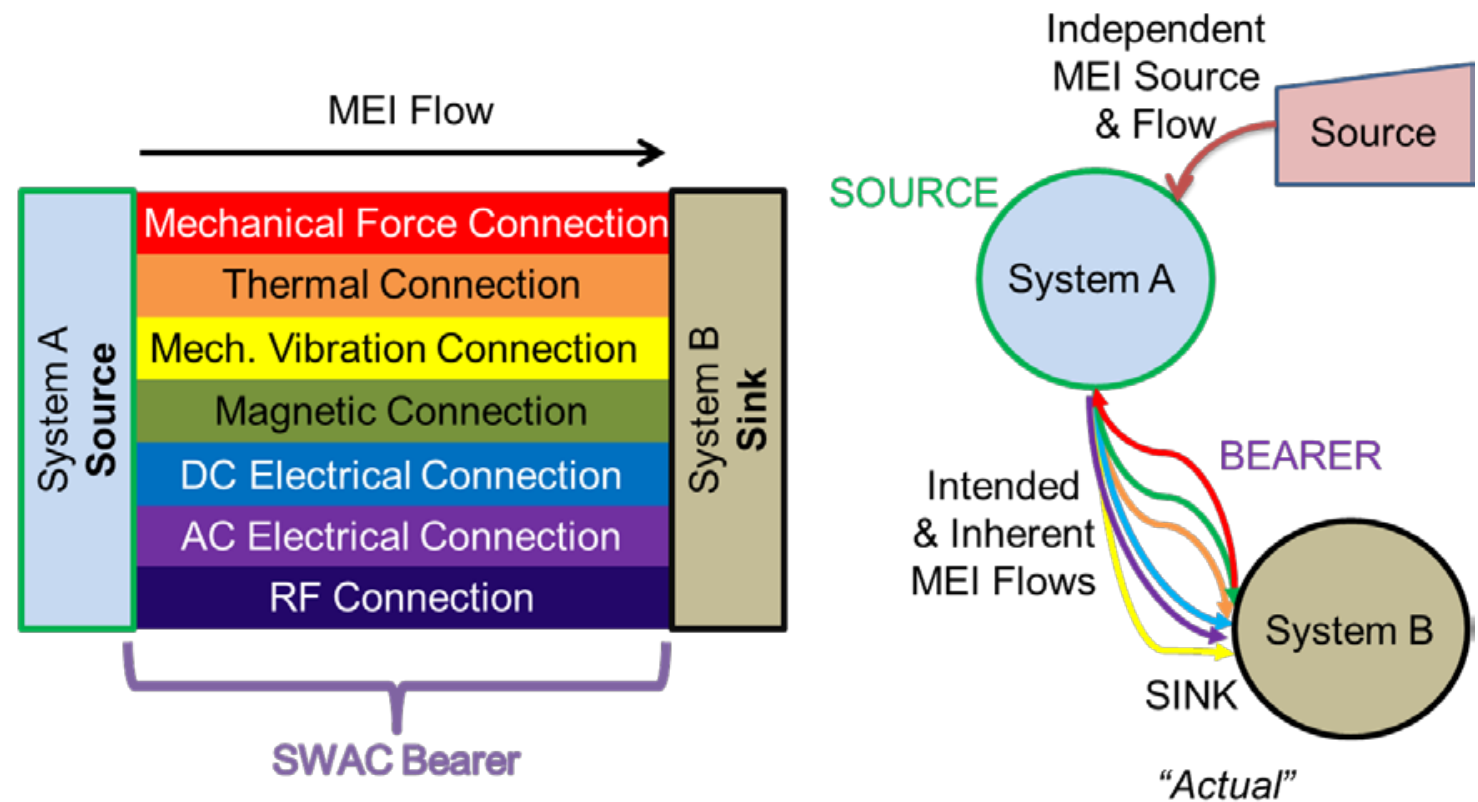

Figure 2: SWA DC power cable and Inherent MEI Transfers

In this case the SWA cables inherent mechanical rigidity (mechanical energy Bearer) of the power cable interfered with the correct operation of the cabinet's shock mounts, and in addition as shown on the right of Figure 2 above the structure of the vessel (mechanical energy Bearer) conducted vibrations from an independent MEI (mechanical energy) source, the vessels propulsion engines, to the radar antenna mast (mechanical energy sink) and degrade the radar's stabilization performance. 
This section has related capability elements to SoS and system constituents and introduced the concept of a systems MEI transfers as consisting of SSB in Intended, Inherent and Independent forms. The section following describes the FFP method employing these concepts.

\section{The "Vee" Model and the FFP Method}

A common representation of the systems engineering process, used for illustrative purposes only here, is the "Vee" diagram shown in Figure 3 below [15]

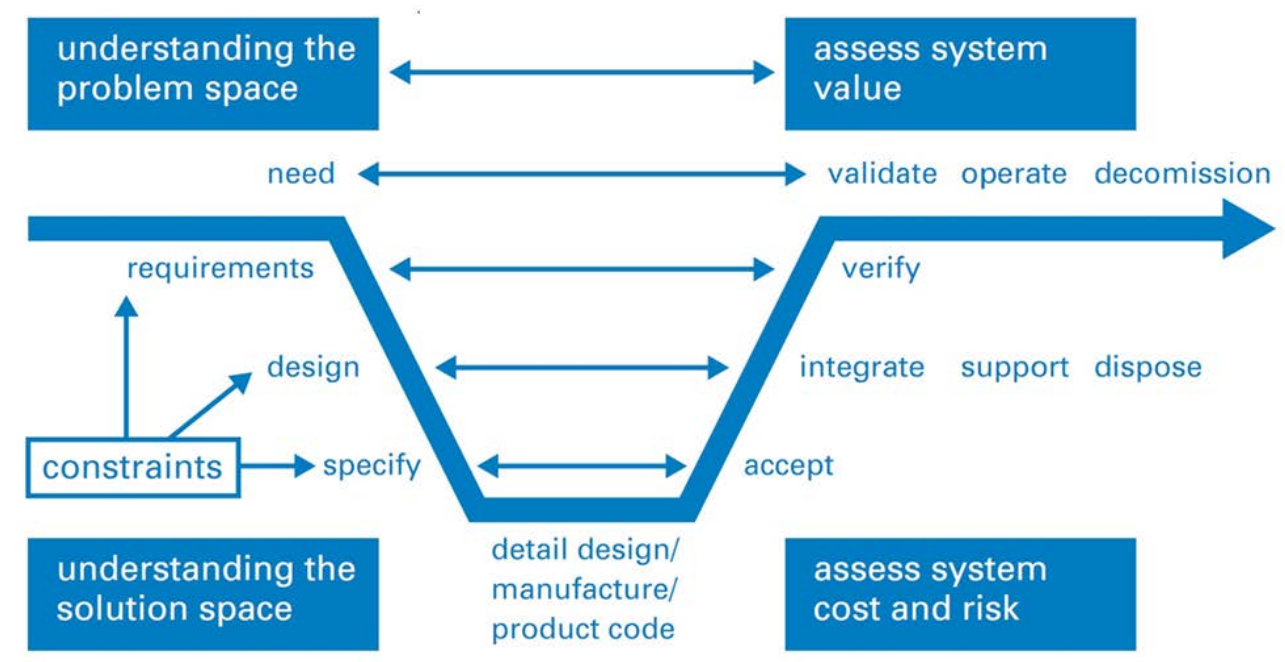

Figure 3. The "Vee" Diagram. A common representation of the systems engineering process, which range, from conceptual models to assist comprehension of complex systems development to detailed product lifecycle and management models.

The Product Lifecycle Management (PLM) artefacts produced at each stage of the "Vee" by different company's implementations are functionally similar, but tailored to their individual needs and constraints.

The FFP method is a transform cascade, as shown in Figure 4 below. The cascade and analyses accommodate SoS constituents that themselves are SoS. Although the cascade below suggests a waterfall process, in practice there is feedback, concurrent development and iteration between the transformations. The data flows in Figure 4 below correlate to the left-hand side of the systems engineering "Vee" model described in the next section.

The method facilitates changes in system capability desired to improve or expand the capabilities of SoS constituent systems to perform system level tasks, as well as those contributing to SoS capability level tasks.

- $\quad$ Transform 1 (Top Left) relates the SoS capabilities in the context of its operational concepts, to the MEI transfers across its boundary that result in the effects that the SoS is desired to have. Mission threads dictate the content and sequence of these MEI transfers producing the desired effects.

- Transform 2 in the cascade is a similar transformation to the first transform, but at the SoS constituent system level.

- $\quad$ Transform 3 groups all the prospective SoS constituent system MEI transfers into a set of system affordances for MEI transfer and identifies the major subsystems of interest. This 
better enables examination and assessment of MEI transfer enhancement from a subsystem viewpoint.

- $\quad$ Transform 4 analyses the affordances for MEI transfer and determines a sub-set as candidates for enhancement, by assessment at the system / subsystem level by the relevant specialist discipline engineers.

- Transform 5 associates system design actions with the system MEI transfer enhancement candidates, guided by the original system design actions and any others that are concurrent with subsystem MEI transfer enhancement.

The bottom-right "PLM Artifacts" represents the Project Lifecycle Management (PLM) documents, engineering drawings, CAD/CAM models etc. into which the enhancement design actions are integrated with other planned actions.

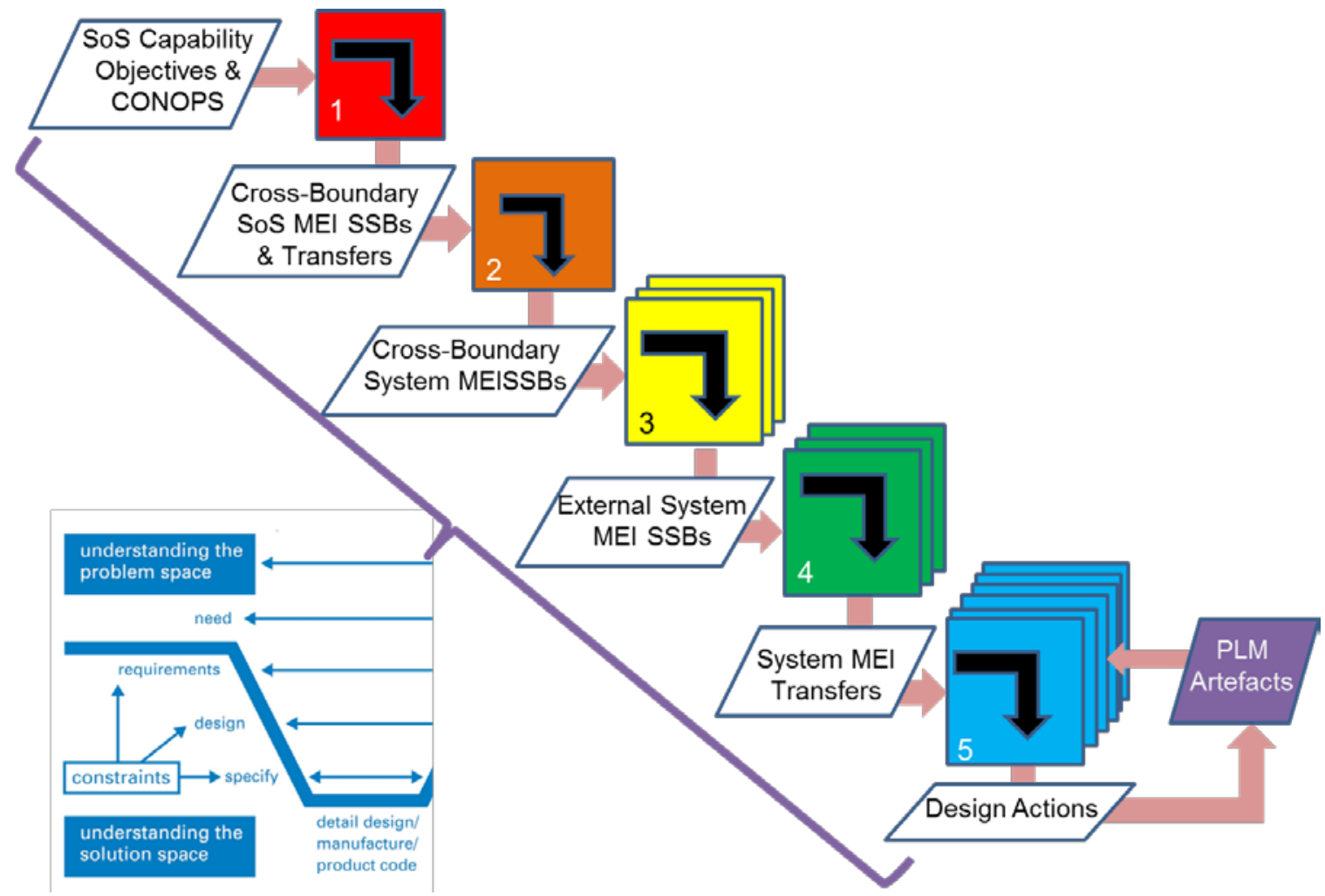

Figure 4. The FFP Method. A cascade of transforms relates top-level SoS capabilities down to constituent systems via MEI Transfers and SSBs to inform MEI enhancement.

Enhancement of system MEI transfer affordances are designed as enablers for new system functionality and SoS capability. Figure 5 below illustrates this and its correlation to the right-hand side of the systems engineering "Vee" model. 


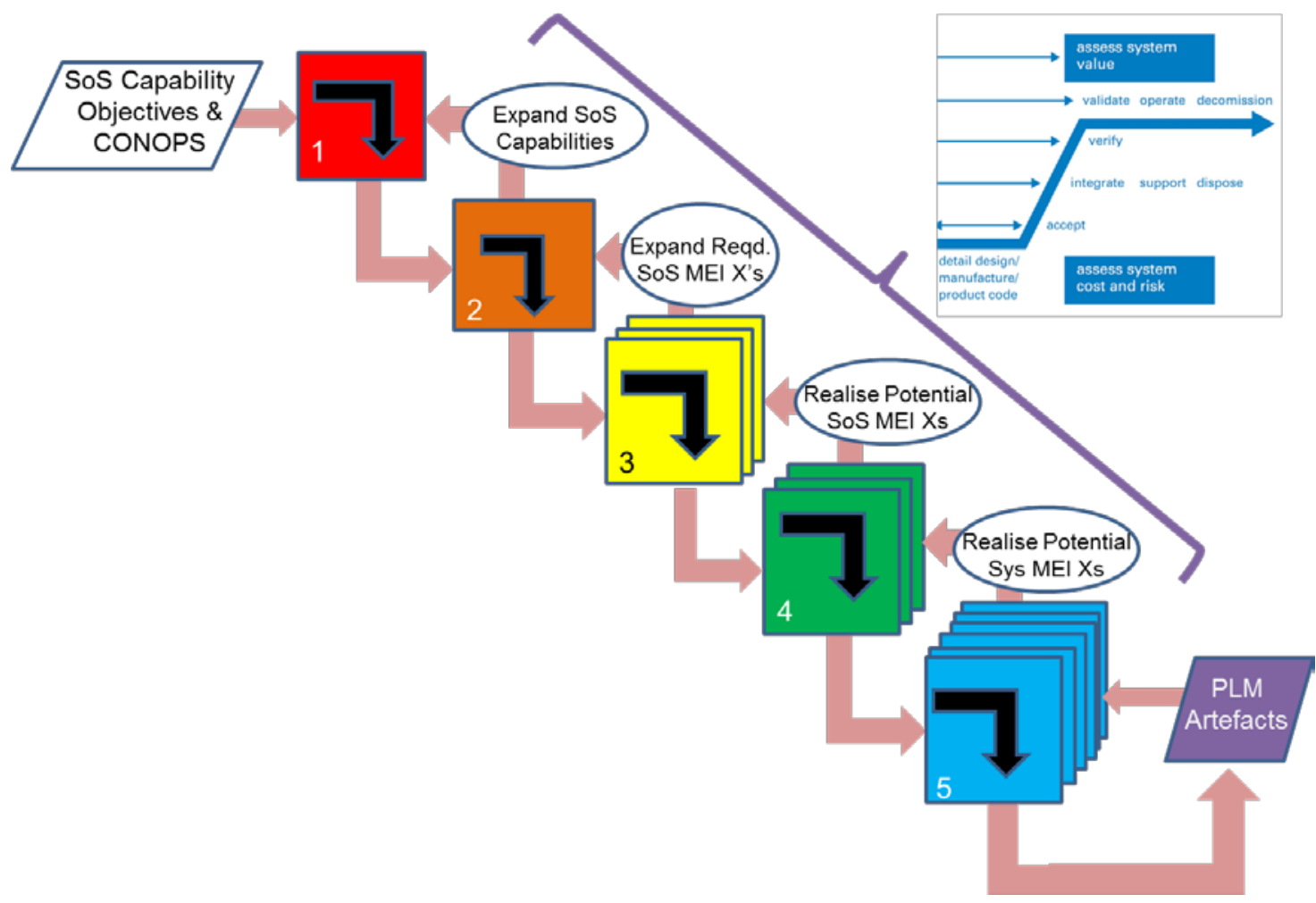

Figure 5. The FFP Method. The cascade of transforms relates low-level MEI Transfers and SSBs up to SoS capabilities via constituent systems.

A MEI transfer enhancement activity could be thought of in terms of its own systems engineering "Vee" model superimposed on the "Vee" model of the system being enhanced.

\section{The FFP Application Process and Technique}

The FFP process is an instantiation of the FFP method that is tailored to the user organization's particular PLM system. The FFP process refers to PLM processes and utilizes PLM project artifacts (e.g. user and system requirements, system design and test specifications etc.) familiar to project staff to reduce the opportunity for error and maintain fidelity with the project / System-of-Interest. In systems oriented engineering companies, the product engineering process and the systems engineering process are often one and the same.

The notion of fitness-for-purpose "maintenance" is used to emphasize that this process can be applied at any stage in the lifecycle shown by the "Vee" diagram in Figure 3 as an opportunity arises. The FFP process is not a new approach to design. It offers a new perspective on projects and the engineering process. Analysis used to populate the matrices identifies opportunities to realize affordances at design opportunities such as scheduled major maintenance intervals, obsolescence resolutions, Mid-Life Improvement (MLI) programs and Improvement through Spares programs.

To assist designers, a three stage analysis technique is suggested. Firstly the designer is asked to identify the Intended ('designed-for') MEI transfers constituent MEI Sources, Sinks and Bearers (SSBs), characterize them in the frequency domain (i.e. identify the bandwidth over 
which they are able to operate) using a cognitive 'seismic to light' sweep, then repeat this cognitive sweep for the Inherent and Independent SSBs that may affect the SoI. From this can be identified MEI SSBs that have the ability to transfer MEI due to their areas of common bandwidth, and hence form Prospective MEI transfers. Secondly an examination of the Prospective MEI transfers in the time domain to promote those with the opportunity to transfer MEI transfer by virtue of their SSB components being active at the same time to Potential MEI transfers. Thirdly the susceptibility of the Potential MEI transfer sinks to MEI conducted to them by the bearers is assessed to determine if any Potential MEI transfer has the capacity to be either problematic or present an exploitation opportunity to usefully enhance the SoIs affordance for MEI transfer.

The architecture and characteristics of the Intended MEI transfers and the characteristics of their constituent MEI SSBs is contained in the product technical data pack (although probably distributed across several engineering disciplines and represented in several different formats) used to manufacture the product. The Inherent and Independent MEI SSBs are likely to be only sporadically captured by a few niche engineering specialities, for example signature management, information architecting, spectrum management / scheduling, and process engineering. A suggestion for bringing these disparate parts together is a MEI meta-model that would provide a reference as part of a product's technical data pack. Figures 3 and 4 below illustrate how such a meta-model provides a more complete view of a system of interest, in this case a SoS with a single input and output.

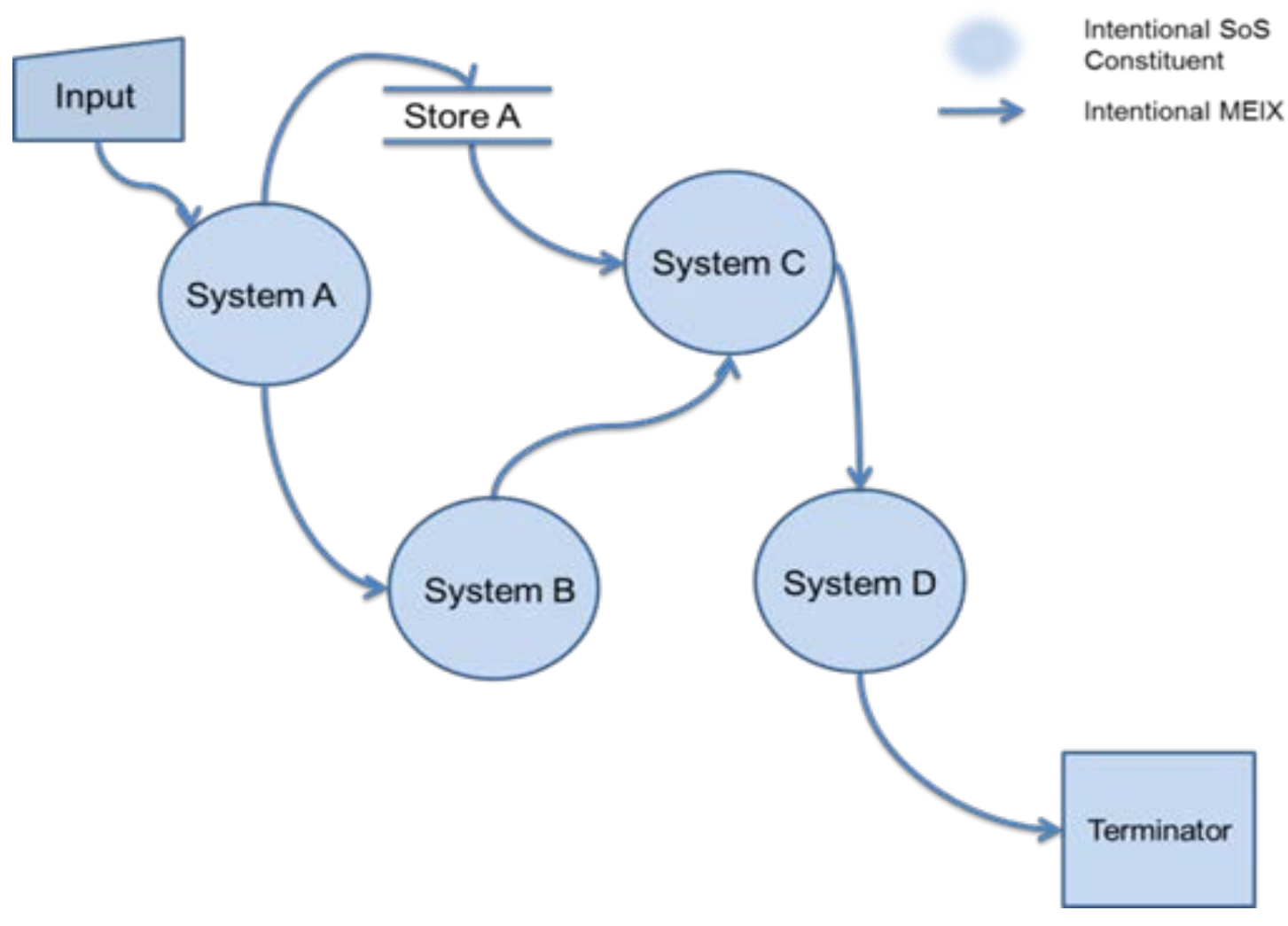

Figure 6. "What we think we have ...": A MEI transfer diagram of a simple System-of-Systems showing the Intended ('designed-for') MEI transfers captured by the technical data pack(s) 


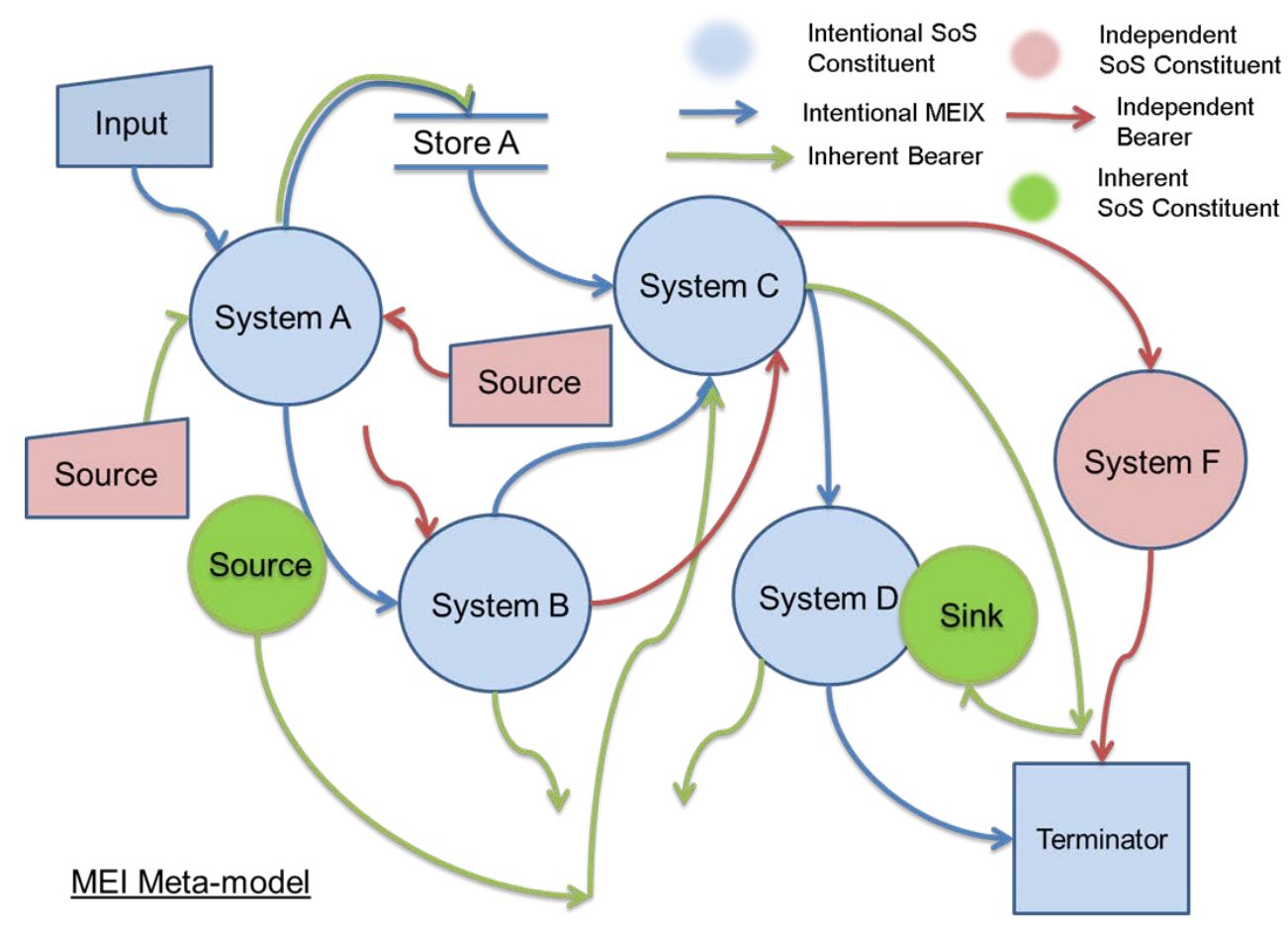

Figure 7. “... and what we actually have”: A MEI transfer diagram of a simple System-of-Systems showing the Intended, Inherent and Independent MEI transfers and SSBs seldom captured or managed.

The vehicle for data capture and visual analytics can be chosen by the FFP adopter, and will be probably be dictated by what application is integrated into their PLM system; this could perhaps be SysML. At the time of writing a simple spreadsheet and open source bioinformatics software platform for visualizing molecular interaction networks has been used.

\section{FFP Salient Points}

Inherent \& Independent MEI transfers almost certainly will not appear in a product's design definition: they will not be controlled or managed, but they may well potentially be either problematic or being utilized by some stakeholders such as users and maintainers and thus will cause problems when they change or are subconsciously withdrawn from the product due to through-life development and modifications. For example, mechanical connections formed by electrical cables to a cabinet may transmit harmful shocks and vibrations to sensitive components within it. Specification compliant replacement components may not have the design margins of original components being exploited by operators and maintainers. One way of encouraging considerations of a system's Intended, Inherent and Independent MEI transfers could be to make a FFP analysis part of a projects systems engineering management plan.

As with systems engineering effort, MEI transfer enhancement may involve some additional cost, and the 'how much is enough' question has to be answered by the practitioners based on what they feel will provide the preferred cost-benefit to their particular case. However it also is an investment for the future which will reduce future implementation risk, reduce operational benefit latency and, by taking the advantage of design opportunities, reduce the overall cost of maintaining fitness-for-purpose by engineering system capability currency to operational needs. MEI transfer enhancement should provide returns similarly to other preparations for the 
future, such as product line architecture reduction, future spares provisioning and "fitted-for-but-not-with" strategies. Commercial arrangements could share risk and benefit between customer and supplier, but the business case has to be assessed on a whole-life basis that includes the cost of upgrades.

The level of provision made for MEI transfer enhancement can be tailored to the needs of the business: it may range from design only, functional models, virtual prototypes, board layout, fitted components to live spares. These provisions could enhance MEI transfer enabling operational augmentation at the system level as well as at the SoS level.

Integration of the FFP method into a supplier's PLM system will benefit the "Creating" system by enhancing it to provide a more complete understanding of "Created" systems by capturing information that previously may have only been tacit. This process also identifies the major subsystems that will be affected by MEI transfer affordance enhancement, and facilitates examination by specialist engineers organized into Work Breakdown Structure subsystem teams that are familiar and experienced in their own areas. FFP embodied into a PLM system can examine a very large data set in a timely manner for inherent and independent MEI transfers than could be problematic or provide opportunities that would be totally impractical otherwise.

An automated FFP process will require the bandwidth, duty and sink susceptibility characteristics of the MEI SSBs in the system definition to be held digitally in the hosting PLM system. These characteristics will exist as component libraries, engineering models and schematics generated by specialist engineering disciplines and systems design artefacts generated by the project system engineers. However, the MEI SSB characteristics may not all be in a machine readable form. The potential industrial exploiter will need to do a trade study between the desired degree of FFP automation and the amount of machine-readable data currently in their PLM system with the work necessary to achieve the level of machine-readable data commensurate with the allocation of function (i.e. either manual, semi-automated or fully automated) that they feel most cost-effectively delivers the desired benefits from incorporating FFP into their engineering processes.

Designers incorporating enhancements into systems enablers for MEI transfers which may be brought into play at some time in the future need to be aware of the capabilities and facilities available to those enabling the enhancement. At first line, close to the point where the SoI is utilized, personnel will have fewer resources than are available at second line (deployable support and repair, field maintenance) and similarly less than those at third line (base workshop).

Any FFP generated design actions need to harmonize with concurrent actions and existing processes and procedures. MEI transfer enhancement and risk mitigation design actions on the selected major subsystems should be incorporated with the company PLM system and able to be integrated with other concurrent design actions, for example those implementing MLIs, carrying out maintenance or repairs.

An FFP goal is to stimulate thought that creates new design actions to realize a potential MEI transfer at the system and hence SoS level to enable a new SoS capability, or mitigate risks that may only be realized when products are fielded. The FFP analysis as appears in this paper is restricted to MEI spectra and duty: individuals may well be stimulated to think of other parameters that could be brought into the project definition to facilitate fitness-for-purpose maintenance. This research is not intended to offer a universal and complete solution; it is a contribution that that provides a more complete view of a system which may well stimulate 
adopters to produce similar analyses tailored to best benefit their individual products and services.

FFP is neither a substitute for knowledge and wisdom, nor a panacea for all ills. FFP adopters may well decide that FFP would not provide an acceptable return-on-investment if applied in areas where nearly all the transfers are of one type, such as data handling and information management or where there is little latitude for modification.

\section{FFP Illustration: Obsolescence Resolution}

This chapter illustrates how an obsolescence recovery exercise provided an opportunity used to enhance a SoS constituent system's affordance for MEI transfer using the method and process described earlier. The system's intended MEI transfers were identified at the transform 4 on the cascade in Figure 4, and extrapolated both up the transform cascade to SoS capabilities and down to subsystem level respectively using requirements and design information in the PLM system. Intended, Inherent and Independent MEI SSBs were identified and characterized, collated into a MEI meta-model, potential MEI transfers identified which were subsequently analyzed for risk and opportunity. Design actions were formulated to reduce risks to an acceptable level and capitalize on opportunities to enhance system functionality and SoS capability as shown in Figure 5.

A Deck Approach Light Projector (DALP). To assist aircraft landing on a carrier, an array of lights on the deck project beams towards the pilot to indicate the movement of the ship and their aircrafts deviation from the ideal approach angle and landing point. A "Wave-Off” (WO) lamp in the array illuminates if it is necessary for an approaching aircraft to abort the landing attempt. Figure 6 below shows a DALP equipment fitted to a carrier.

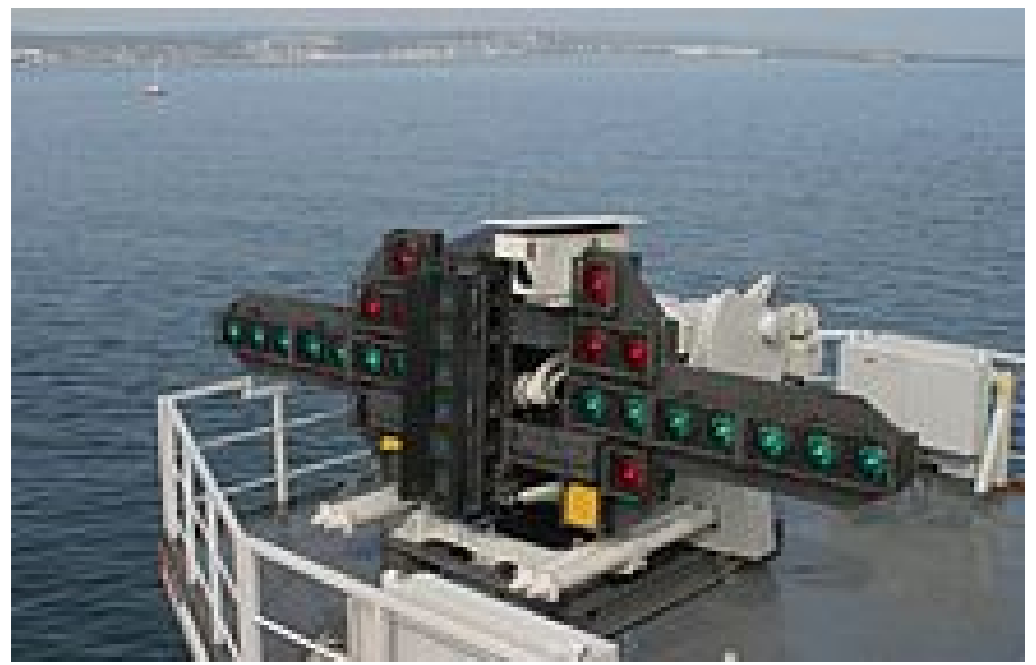

Figure 6. A Deck Approach Light Projector mounted on a naval vessel.

Obsolescence of some of the original equipment filament bulbs provided an opportunity to reduce downtime and maintenance cost by capitalizing on advances in LED technology. No explicit enhancements to the DALP equipment have been requested at the time of the obsolescence resolution exercise, i.e. not in response to a function or performance upgrade requirement; however a FFP analysis shows an opportunity to enhance of the DALP's ability to transfer MEI in parallel with obsolescence resolution at little extra cost.

MEI Transfer Affordance Opportunity. The DALP WO MEI transfer affordance is a lamp that when flashed at $1 \mathrm{~Hz}$. instructs the pilot of an approaching aircraft attempting to land to 
abort the landing. The FFP method analysis determines the potential for MEI transfer enhancement of the DALP's MEI affordances and SSBs, and identifies the potential of the WO affordance for enhanced information transfer.

The on/off and off/on response time of a LED is much faster than that of a conventional incandescent lamp which requires a finite time to heat up and cool down, so the faster switching characteristic of a LED lamp could provide an opportunity to enhance information transfer of the WO affordance, by modulating its output. The enhanced WO affordance would provide the carrier an available, non-broadcast, secure, low-latency and jam-resistant communications link to an approaching aircraft.

The new LED lamps require new driver circuits which are controlled by the DALP data bus. (Note: circuit is for illustration purposes only, and the data bus connections are not shown). The new circuitry required for the new LED lamps is shown on the left-hand side of Figure 7 below.

The new LED WO lamp driver circuit has a modulation input added to it. The modification to the new WO lamp driver circuitry is shown circled on the right-hand side of Figure 7 below.
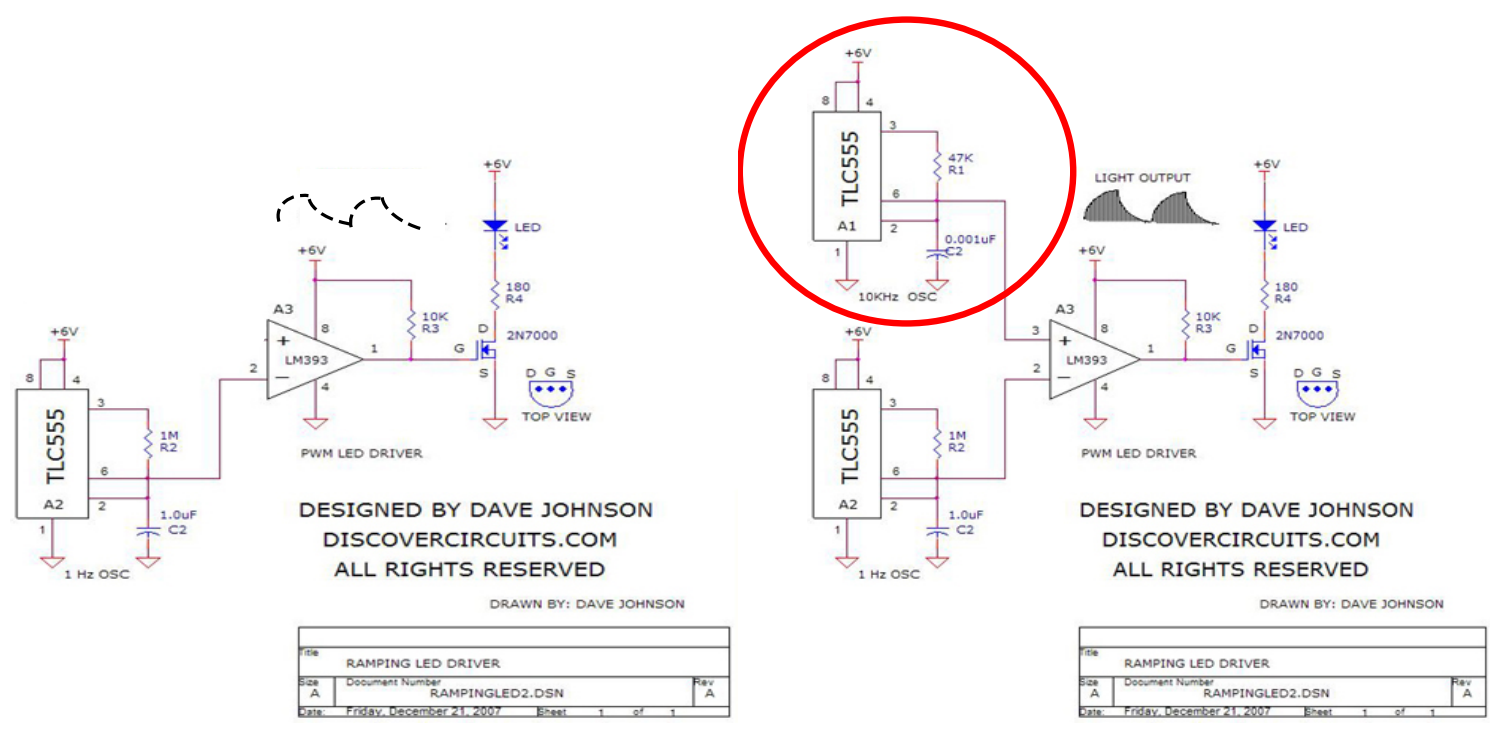

Figure 7: Illustrative LED lamp driver circuit and WO lamp driver circuit enhancement

The MEI transfer (in this case Information) enhancement facilitates a new, secure, un-jam-able data transmission from the carrier to an approaching aircraft. The carrier houses extra functionality not occupying processor and memory space on the aircraft where it is at more of a premium.

DALP Enhancement Considerations. The DALP enhancement costs are small, as in this instance the equipment and associated documentation are already scheduled to be modified, and the number of DALPs in-service is small. The enhancement above does not significantly increase component count and type, and in this case should not significantly impact costs from bought-out materials, testing, equipment support publications, training etc. The WO enhancement modification is incorporated into the design actions forming part of the DALP project plans and documentation in accordance with the company lifecycle management process. 
FFP analysis exploiters need to decide if there is merit at a design opportunity such as the DALP obsolescence recovery task in analyzing both the "before" and "after" situations. In the case of this obsolescence recovery task the move to LED lamps reduced the WO lamp energy transfer by a significant reduction in the Infra-Red (IR) region. This meant that although the new lamps retained the Night Vision Goggle (NVG) and Forward-Looking Infra-Red (FLIR) visibility of the old lamps, the inherent IR energy transfer of the LED lamps was insufficient to prevent ice build-up in artic conditions, and a de-icing provision had to be made that was fulfilled by the inherent IR output of the old filament lamps.

DALP MEI transfer Affordance Enhancement Exploitation. The carrier is a central part of an SoS (a carrier group of vessels) that is conducting military operations in a littoral scenario, whose purpose is to provide Intelligence/Surveillance/Target Acquisition and Reconnaissance (ISTAR) capability to friendly forces ashore. An unforeseen change in the political situation around the carrier groups operations meant that some military tasks being achieved by manned aircraft became untenable. As a consequence of the external change the carrier group was no longer fit for the purpose of providing an ISTAR capability.

Candidate solutions were examined to determine their feasibility, impact and timeliness on both the problem and the capabilities available from the SoS resource, which included the necessary changes and enhancements to the MEI transfers. A preferred solution of Unmanned Air Vehicle (UAV) operations was chosen from a candidate set. Available UAVs have a core capability of operating from land, but do not have the ability to operate from a carrier. The UAV's are normally landed by a human pilot under remote control, but the latency in the control loop is too large to enable the remote pilot to compensate and make adjustments for the movements of the carrier at sea. The enhanced carrier to aircraft information transfer via the light projector is exploited to provide a command link to an unmanned aircraft (UAV) via its panoramic IR/TI camera, auto-tracker and flight control system to provide a low-latency minor control loop to relieve the pilot of compensating for the movements of the vessel, enabling him to apply the flight commands to the UAV landing on the carrier much as he would do for a landing on the ground.

The provision made by the supplier during the DALP obsolescence recovery task was brought on-line whilst the carrier group was on-station, and enabled the available UAVs to be operated from the carrier, thus maintaining fulfilment of the necessary ISTAR tasks without placing pilots, and expensive aircraft containing sensitive intellectual property in harm's way.

\section{Conclusion}

The interconnectivity and concomitant complexity of systems is rapidly increasing, meaning that engineers must now think in terms of the fitness of purpose of systems of systems, as opposed to single systems. The FFP method is offered as an holistic-thinking approach that will assist engineers identify concealed MEI sources, sinks, bearers and transfers not otherwise included in SoS/System definition which may lead to unexpected emergent phenomena either problematic and only revealed "late in the day", or being employed in-service unbeknown and not under the control of the design authority. Examples of such emergence in both naval and land-based domains have been found during the test and development of this work. The more complete insight from this approach enhances delivered products / services, and also improves the PLM engineering processes used to create them, and facilitates the task of identifying and implementing SoS adaptation (through reconfiguration at the SoS level or changes to constituent systems) in order to maintain that SoS as fit-for-purpose. This paper has provided an overview of the FFP method and indicated how it may be implemented in organizational 
processes such that it may offer improved management of a complex SoS, enabling it to be maintained as Fit For Purpose to address new unforeseeable tasks and/or changes, both internal and external, throughout the lifecycle at an affordable scale.

\section{References}

[1] Norman, D.A., 1999. 'Affordance, Conventions and Design'. Association of COmputing Machinery: Interactions, (May-June), pp.38-42. Available at: http://www.jnd.org/dn.mss/affordance_conventi.html.

[2] Maier, M.W. (1998), 'Architecting Principles for Systems-of-Systems', Systems Engineering, Vol. 1, No. 4, pp. 267-284.

[3] Hopkin, P., 2002. Holistic Risk Management in Practice 1st ed., London: Witherby \& Co. Ltd. p.3.

[4] Rumsfeld, D., 2002. US DoD briefing to NATO, Brussels. NATO Press Conference, p. 8. Available at: http://www.nato.int/docu/speech/2002/s020606g.htm.

[5] De Meyer, A., Lock, C.H., Pich, M.T. (2002) Managing Project Uncertainty: From Variation to Chaos, MIT Sloan Management Review, 43(2).

[6] Ring, J, 2012. INSIGHT, July 2012 p.42 INCOSE

[7] Ring, J., Tenorio, T., 2012. INSIGHT Volume 12 Issue 2 (July) p.11 INCOSE

[8] Smith, R., 2005. The Utility of Force 1st ed., London: Pengiun. p.3.

[9] Ashby, W.R., 1956. An Introduction To Cybernetics, London: Chapman \& Hall Ltd. p.202-208. Available at: http://pespmc1.vub.ac.be/books/IntroCyb.pdf.

[10] Boardman, J. \& Sauser, B., 2006. 'System of Systems - the meaning of “of.”. 2006 IEEE/SMC International Conference on System of Systems Engineering Los Angeles, CA, USA - April 2006. Los Angeles, pp. 118-123.

[11] Dalton, S., 2013. The 21st Century Character of Air Power. , pp.1-11 2013 Sir Sydney Camm Lecture.pdf.

[12] UK MoD. (2008). UK MoD AOF. Aquisition Operating Framework. Retrieved from https://www.gov.uk/acquisition-operating-framework

[13] Burrowes, D. and Squair, "Managing the Emergent Properties of a Design," Proceedings of the 9th International INCOSE Symposium, Brighton, UK, 1999

[14] Sillitto, H. (2011). 'Integrating systems thinking, systems science and systems engineering - understanding the difference and exploiting the synergies'. Presentation at INCOSE ASEC 2011 (p. 20), INCOSE.

[15] INCOSE, 2009. Z1 Guide, Revision 3, p.2. Available at: http://incoseonline.org.uk/Program_Files/Publications/zGuides_1.aspx?CatID=Public ations. 


\section{Biographies}

Steve Hinsley is a mature $\mathrm{PhD}$ research student under Professor Mike Henshaw and Professor Carys Siemieniuch in the Engineering Systems-of-Systems Group of the School of Electronic, Electrical and Systems Engineering at Loughborough University. The research is funded by Loughborough University Graduate School and supported by Thales.

Previously Steve was technical lead of the systems engineering capability for BAE Systems Advanced Technology Centre, and Chief Engineer at the

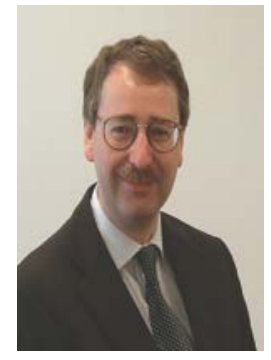
Systems Engineering Innovation Centre. He is a Chartered Engineer, a member of the IET and INCOSE. He holds a BSc. (Hons.) in Electrical Engineering from De Montfort University Leicester and a MSc. in Advanced Systems Engineering from Loughborough University.

Michael Henshaw is Professor of Systems Engineering and leads the Engineering Systems of Systems (EsoS) Research Group. His research focuses on integration and management of complex socio-technical systems, with a particular emphasis on the challenges of through-life management of systems and capabilities.

Professor Henshaw graduated in applied physics, and his early research focused on laser-plasma interactions, using computational fluid dynamics to investigate various phenomena in applications such as X-ray lasers. He joined

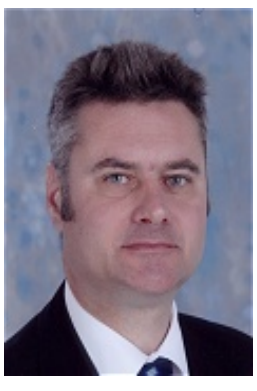
British Aerospace (later BAE Systems) as an aerodynamicist and worked for seventeen years in aeronautical engineering tackling problems associated with unsteady aerodynamics (computational and experimental) and, later, multi-disciplinary integration. He was appointed to a chair in Systems Engineering at Loughborough in 2006 to direct the large multi-university, multi-disciplinary program in Network Enabled Capability.

Professor Siemieniuch is a Professor of Enterprise Systems Engineering and a member of the Engineering Systems of Systems Research Group in the Department. She is a Chartered Ergonomist and Human Factors Specialist with expertise across the full range of systems-related human factors topics. Her key skills are in knowledge lifecycle management systems, organizational and cultural aspects of enterprise modeling techniques, organizational systems architectures, dynamic allocation of function and the design of complex systems. She is active in both the military and civilian

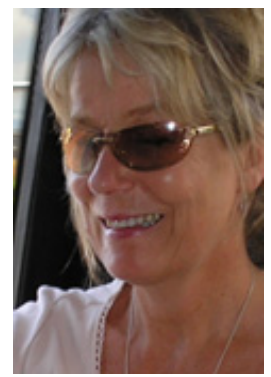
domains. 